\title{
Effect of Agitation Work on Heat Transfer during Cooling in Oil ISORAPID 277HM
}

\author{
Bohumil Taraba* - Steven Duehring - Ján Španielka - Štefan Hajdu \\ Slovak University of Technology in Bratislava, Faculty of Materials Science and Technology, \\ Institute of Production Systems and Applied Mechanics, Slovak Republic
}

\begin{abstract}
The article focuses on the issue of heat treatment. The cooling curves were obtained for Isorapid 277HM with an experimental way of temperature measuring and their statistical processing. Experimental method was consistent with the test normative ISO standard 9950 (Wolfson's test). The cooling oil Isorapid $277 \mathrm{HM}$ was agitated with different agitation work and had a constant temperature of $50{ }^{\circ} \mathrm{C}$. In the next part of this article the surface temperature depended combined heat transfers were calculated. The methodology was based on inverse heat transfer. The interpretation code was software ANSYS and ORIGIN.
\end{abstract}

Keywords: quenching, cooling curve, agitated oil, heat transfer, Wolfson's probe, ANSYS

\section{O INTRODUCTION}

Heat treatment is a multiparameters process. The selection of appropriate parameters predicts required behaviours of treated components. The kind of quenching medium, the selection of quenching medium temperature and the selection of the medium state (unagitated, agitated) are determining factors. Quenching oil Isorapid 277HM belongs to cooling oils common in use. A prediction of treated components behaviour during a cooling process is possible only in the case if the boundary conditions of the process are defined. Before the application of a cooling process numerical simulation, the heat transfer coefficient on the component surface should be defined quantitatively. The experiment, applying simulation model and numerical solution, is able to test the influence of heat treatment parameters on an immediate and final state of a component. A cooling curve is the basis for determining the combined heat transfer coefficient (HTC) as a function of temperature. The current situation presents two ways of getting the HTC cooling curve: direct and inverse approach. Direct access is represented in the publication [1]. HTC is obtained by calculating based on the classical theory of heat conduction in infinite long cylinder with small Biot's number $(B i<0.1)$ in few simple recursive computations using the "Heat Transfer Coefficient Wizard". The comparison between measured cooling curves (derived cooling rate curve) with calculated curves is only visual.

The heat transfer coefficient inverse method is based on iterative approach loading simulation model in the form of $H T C$ and the effect of temperature at thermocouple (TC-temperature) [2] to [4]. The inverse numerical method is implemented in the software
SQintegra also. This program is used as the evaluation tool of the IvfSmartQuench instrument [2].
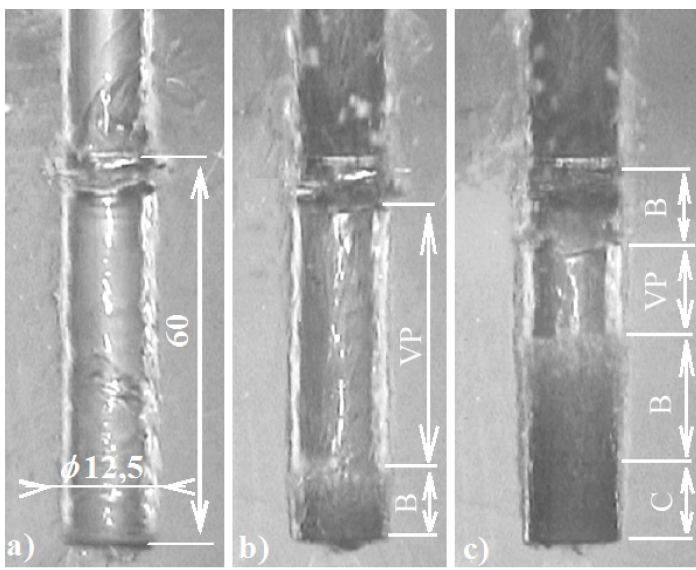

Fig. 1. ISO probe cooling process in Isomax 166, a) vapour blanket (VP) in time $1 \mathrm{~s}, b$ ) begin of boiling (B) in time $2.8 \mathrm{~s}, \mathrm{c}$ ) boiling (B) and convection (C) in time $6.3 \mathrm{~s}$

Inverse-numerical-correlation method (INC) defines HTC over inverse heat transfer problem, which was proposed by the authors of this article. The INC method is applied to the solution of direct well-posed inverse problems. Through the controlled iterative process a result which is very likely and useful for computer prediction of thermal treatment processes can be found. The main active part of this procedure is a researcher with theoretical knowledge and experiences with numerical analyses. Typical for the inverse methods is that there exist an infinite set of solutions in general. Only the right setting of statistical criteria get the result with the high degree of reality. Fig. 1 shows the cooling process of ISO probe in optically transparent quenching oil Isomax 166. Photographs in Fig. 1 show that the cooling process 
in the three forms of heat transfers (radiation, boiling and convection) are a continuous process without step change (photo from article authors). Then, the determinate $H T C$ must also be continuous.

The methodology and results of a cooling effect quantification of oil Isorapid $277 \mathrm{HM}$ with chosen agitation work at temperature $50{ }^{\circ} \mathrm{C}$ are presented in the article.

\section{EXPERIMENTAL}

Quenching oils ISORAPID are accelerated quenching oils with very good evaporation stability and fast quenching properties. These oils have been especially designed for an application in sealed quench furnaces. They ensure rapid and homogeneous cooling of all parts during batch quenching and also rapid decay of the vapour blanket within the batch. Their application in open quench baths reduces smoke and flame formation significantly [5].

The experimental set-up in Fig. 2 consisted of electrical resistance furnace of LM 212.10 type, cylinder-shaped experimental probe (Table 1, Fig. 3), oil Isorapid $277 \mathrm{HM}$ with a mass of $28 \mathrm{~kg}$, portable USB-based DAQ for thermocouples NI USB 9211 for a digital record of measured temperatures, frequency converter MICROMASTER 440 (MM440), a personal computer and pneumatic manipulator for probe moving. A material of probe was austenitic stainless steel DIN 1.4841 with high temperature resistance. Thermophysical material properties were obtained from experimental measuring by NETZSCH apparatus: LFA 427, DSC $404 \mathrm{C}$ Pegasus and Dilatometer $402 \mathrm{C}$.

Geometrical and initial conditions of the experiment were based on the quenching test ISO 9950 [6]. Before cooling, the probe was heated to the initial temperature of $850{ }^{\circ} \mathrm{C}$. The temperatures were measured by the standard 304SS thermocouple of $\mathrm{K}$ type with diameter of $1.53 \mathrm{~mm}$ located in the centre of the probe. Temperatures were recorded 5 times per second. A set of measurements was repeated six times for each state of oil. Each set of measured cooling curves was averaged into a core cooling curve. There were seven oil states realized, one for unagitated and six for agitated. Temperature measurement started from the moment when the centre of gravity of probe reached the oil level.

Power parameters (torque moment and input $\mathrm{rpm}$ ) of the swirl devices were obtained from the data of frequency converter MM440.
Table 1. Thermophysical material properties of austenitic stainless steel DIN 1.4841

\begin{tabular}{cccc}
\hline$T\left[{ }^{\circ} \mathrm{C}\right]$ & $\lambda\left[\mathrm{W} \cdot \mathrm{m}^{-1} \cdot \mathrm{K}-1\right]$ & $\rho\left[\mathrm{kg} \cdot \mathrm{m}^{-3}\right]$ & $\mathrm{c}_{\mathrm{p}}\left[\mathrm{J} \cdot \mathrm{kg}^{-1} \cdot \mathrm{K}^{-1}\right]$ \\
\hline 0 & 13.5 & 7880 & 474 \\
100 & 15.0 & 7854 & 490 \\
200 & 16.8 & 7814 & 512 \\
300 & 18.6 & 7773 & 525 \\
400 & 20.0 & 7731 & 535 \\
500 & 21.3 & 7689 & 544 \\
600 & 23.2 & 7645 & 569 \\
700 & 24.8 & 7601 & 581 \\
800 & 25.6 & 7556 & 589 \\
900 & 27.1 & 7511 & 600 \\
\hline
\end{tabular}

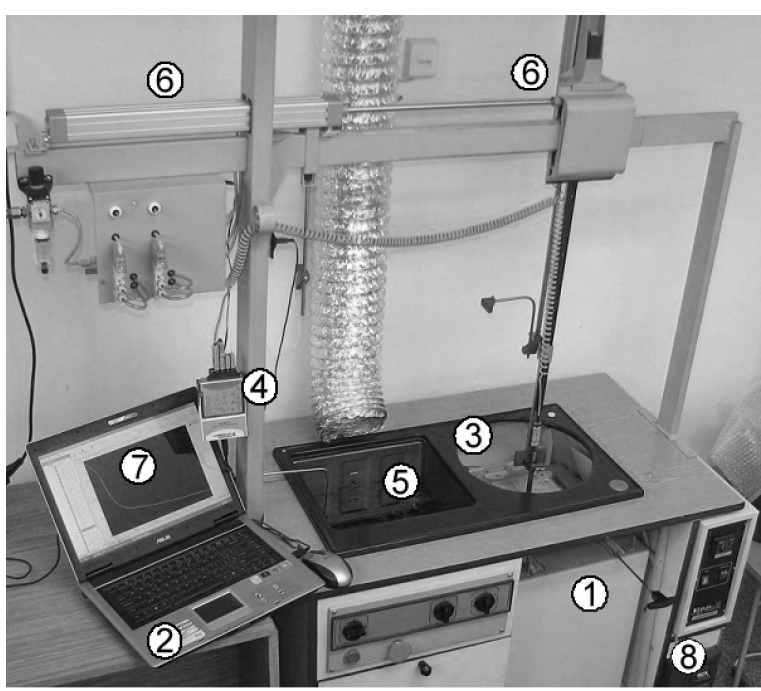

Fig. 2. Experimental setup: 1- electrical resis-tant furnace, 2- personal computer, 3 - probe with a thermocouple, 4 - NI USB 9211 converter, 5 - cooling medium and its heater, 6 - pneumatic manipulator, 7- record of cooling curve, 8-frequency inverter

\section{THEORETICAL BASE OF THE TASK}

Transient temperature field $T=T(r, z, t)$ of a cooled probe is described by Fourier-Kirchhoff differential equation (FKDE) of heat conduction for cylindrical coordinate system [8],

$$
\frac{\partial T}{\partial t}=\frac{\lambda(T)}{\rho(T) c_{\mathrm{p}}(T)}\left[\frac{\partial^{2} T}{\partial r^{2}}-\frac{1}{r} \frac{\partial T}{\partial r}+\frac{\partial^{2} T}{\partial z^{2}}\right]\left[\mathrm{K} \cdot \mathrm{s}^{-1}\right],
$$

where $\lambda(T)$ is coefficient of heat conductivity, $\rho(T)$ density, $c_{\mathrm{p}}(T)$ specific heat, $r$ radius $[\mathrm{m}]$ and $z$ is height of probe $[\mathrm{m}]$.

Combined heat transfer coefficient $\operatorname{HTC}\left(T_{\mathrm{s}}\right)$ was determined as the function of the probe surface temperature $T_{\mathrm{S}}$ for constant oil temperature $T_{\mathrm{r}}$. The 
condition of equality of heat flux is valid on the probe surface in time point $t_{\mathrm{i}}$ by formula [4]:

$$
-\left.\lambda(T) \operatorname{grad} T\right|_{t_{i}}=\operatorname{HTC}\left(T_{s}\right)\left[T_{s}\left(t_{i}\right)-T_{r}\right] .
$$

Other assumptions of thermal tasks: the probe material is isotropic and its thermophysical properties are temperature dependent, the cooling process is isobaric, the temperature field is not dependent on the angle $\varphi, T \neq f(\varphi)$, coolant temperature is constant throughout the process, $T_{\mathrm{r}} \neq f(t)$. Heat generation in unit volume per unit time was not take account because in probe material there are no phase transformations in the temperature interval 50 to $850^{\circ} \mathrm{C}$.

A thermal task is solved by the finite element method (FEM). The FEM solution procedure is in the form of equation:

$$
\mathbf{K}_{1} \cdot \mathbf{T}+\mathbf{K}_{2} \cdot \mathbf{T}+\mathbf{K}_{3} \cdot \dot{\mathbf{T}}-\mathbf{P}=\mathbf{0},
$$

where $\mathbf{K}_{1}$ is heat conduction matrix, $\mathbf{K}_{2}$ matrix of boundary conditions, $\mathbf{K}_{3}$ enthalpy matrix, $\mathbf{T}$ temperature vector, $\dot{\mathbf{T}}$ time derivation of temperature and $\mathbf{P}$ is vector of outer loads.

Absolute value of relative error $\delta_{T}$ was obtained by formula:

$$
\left.\delta_{T}\right|_{t_{i}}=\left|\frac{T_{T C}-T_{\text {ans }}}{T_{T C}}\right| \cdot 100,
$$

where $T_{T C}$ is measured temperature and $T_{a n s}$ is temperature of numerical solution, both for time $t_{i}$.

Input power into oil per $1 \mathrm{~kg} P_{w}$ was calculated from torque moment and angular velocity values at device for swirling by formula:

$$
P_{w}=\frac{2 \pi M_{\tau} n}{m},
$$

where $M_{\tau}$ is torque moment, $n$ rotational speed and $m$ is mass of oil in device.

\section{NUMERICAL SIMULATION}

Engineering-scientific program code ANSYS [7] was the interpretation program of numerical simulation. Geometrical model of the probe was the lower half part of the cylinder, Fig. 3.

Applied elements were axisymmetric with linear base function and surface temperature behaviour option. Surface temperature behaviour allows the application of thermal load $\operatorname{HTC}\left(T_{\mathrm{s}}\right)$ as the actual surface temperature function. The generated mesh was mapped with the length of the element edge $0.25 \mathrm{~mm}$. Calculation procedure was transient and nonlinear. Time step was $0.01 \mathrm{~s}$.
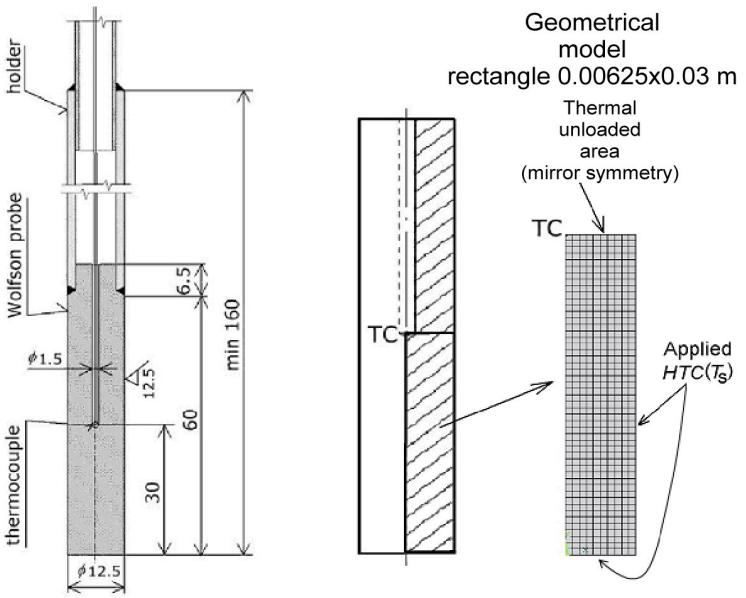

Fig. 3. Probe geometry and geometrical model with generated mesh

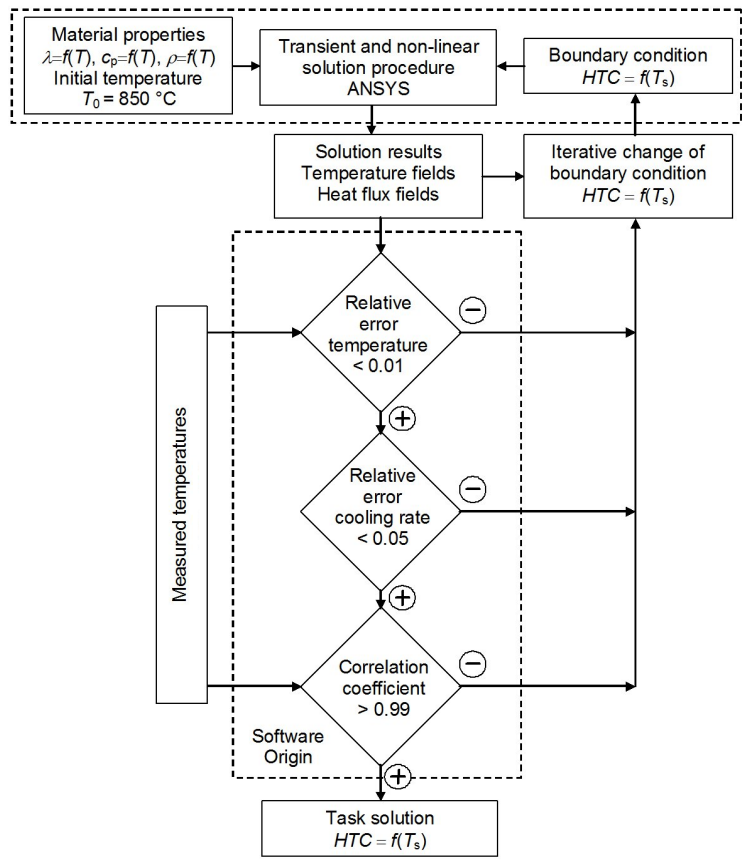

Fig. 4. Block diagram of iterative solution of the boundary condition - INC method

Through the solution of simulation model of thermal nonlinear and transient task in the ANSYS the temperature curve for chosen $H T C$-loads values was found. Then, a comparison with measurement temperature curve followed and the process was repeated. The curve fitting takes account of the temperature and cooling rate curve. Task solution by the INC method must meet the following criteria: absolute value of relative error for measured and calculated temperature in the $i$-time must be less 
than $1 \%$, absolute value of relative error for cooling rates derived of measured and calculated temperature must be less than $5 \%$ and the correlation coefficient between the measured and calculated temperatures in the cooling time must be greater than 0.99. Block diagram of iterative solution of the boundary condition - INC method is showed in Fig. 4.

\section{OBTAINED RESULTS}

Time dependences of 7 measured temperatures during probe cooling from $850{ }^{\circ} \mathrm{C}$ into unagitated and agitated oil at temperature $50{ }^{\circ} \mathrm{C}$ are shown in Fig. 5. These core cooling curves were the basis for INC method applying.

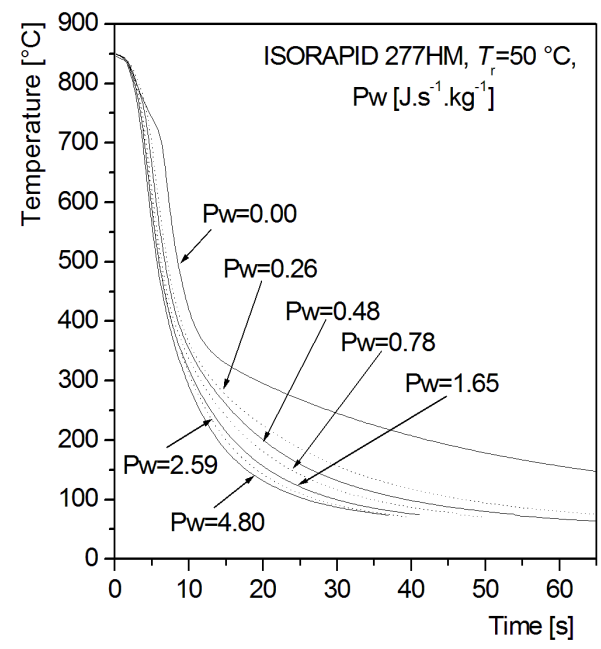

Fig. 5. Set of measured temperatures, unagitated and agitated oil ISORAPID 277HM

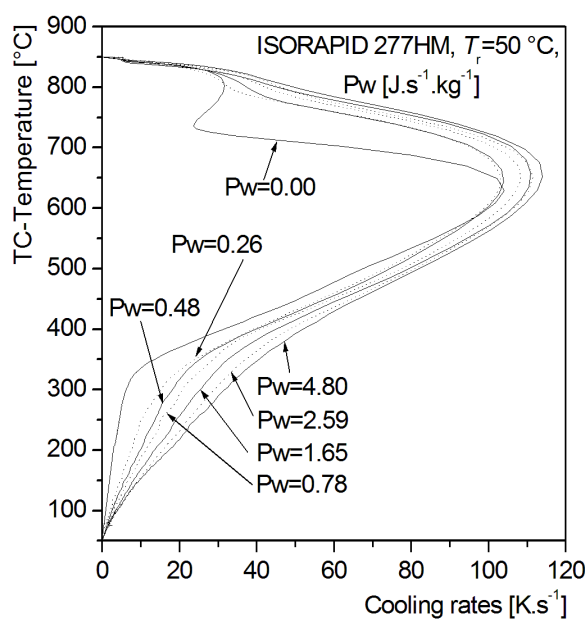

Fig. 6. Cooling rates for unagitated and agitated oil ISORAPID $277 \mathrm{HM}$
In Fig. 6 are plotted cooling rates curves (derived from core cooling curves).

There is a distinct difference between cooling in unagitated and agitated oil. The lowest value of cooling rate is for unagitated oil and with energy supplied into oil increases the cooling rate and temperature at the centre at which the maximum cooling rate. The cooling rate interval is of 103 to 114 $\mathrm{K} \cdot \mathrm{s}^{-1}$.

Combined heat transfer coefficient dependences of probe surface temperatures for unagitated and agitated oil are the main results of INC method and are shown in Fig. 7.

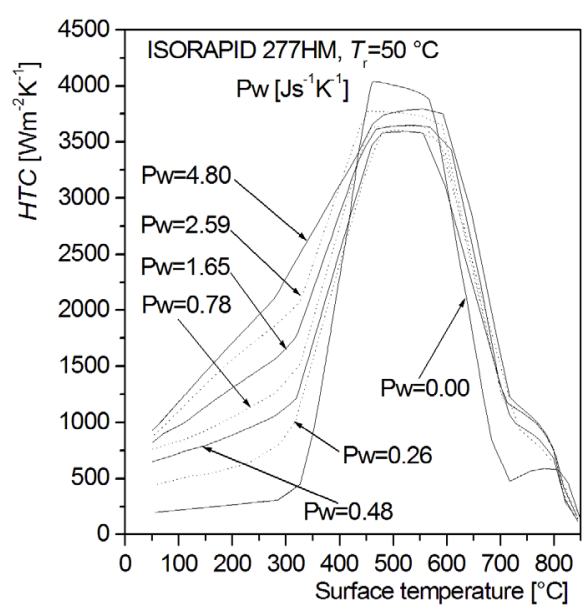

Fig. 7. HTC curves for unagitated and agitated oil ISORAPID $277 \mathrm{HM}$

Forced movement of Isorapid 277HM oil alters the cooling process of probe in the vapour phase. The existence of vapour phase is shorter at higher surface temperatures and $H T C$ reaches higher values than in the case of unagitated oil also. An important feature is knowing that the effect of agitation of oil will be reflected most in the convection heat transfer surface temperature below $317{ }^{\circ} \mathrm{C}$. HTC varies with the size of the energy supplied into oil. $H T C$ values are readable from Fig. 7.

Combination of experimental cooling curves and numerical simulation using INC method gives the values of absolute value of relative errors that are showed in Fig. 8.

For the purposes of clarity Fig. 8 has been selected four values of energy supplied into oil. The absolute relative error between the measured and calculated values of cooling curve was evaluated. The maximum value was $1.09 \%$ and the average error for all cases was less than $0.45 \%$. 


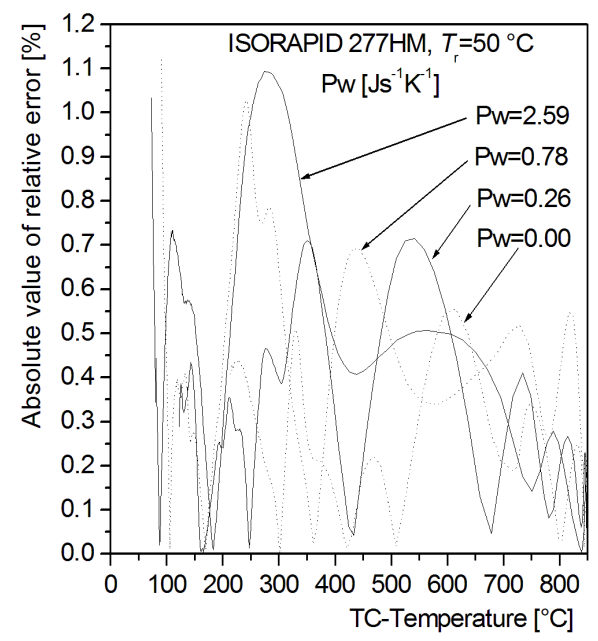

Fig. 8. Absolute values of relative errors for core cooling curve fitting for chosen energy input

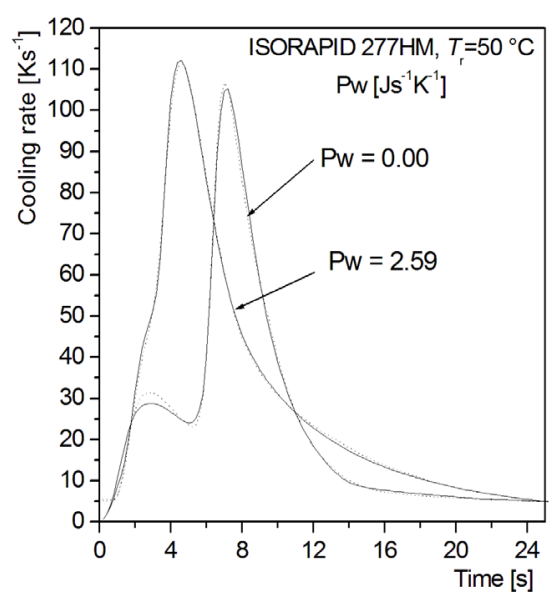

Fig. 9. The fitting comparison for cooling rate curves for unagitated oil and agitated oil with energy input $2.59 \mathrm{~J} \cdot \mathrm{s}^{-1} \cdot \mathrm{kg}^{-1}$

The temperature curve fitting was then very close and it was not possible to graphically represent both curves. The correlation coefficient between calculated and measured temperatures was obtained 0.9998 for all solved cases.

The test was made for a relative error between the cooling rate obtained from the measured cooling curve and the curve of the INC method also. Fig. 9 shows two selected cooling rate curves for unagitated and agitated oil with energy input $2.59 \mathrm{~J} \cdot \mathrm{s}^{-1} \cdot \mathrm{kg}^{-1}$. For unagitated oil average absolute value of relative error was 0.23 and for energy input was the calculated error $2.02 \%$.

\section{CONCLUSION}

A definition of heat transfer from the probe into the coolant as the inverse problem of heat conduction using a suitably selected control parameters is an appropriate method to quantify the $H T C$ under different conditions. Energy input into agitated oil in the form of work per $1 \mathrm{~kg}$ media allows the reproducibility of the experiment. The obtained HTC are properties of the tested oil and are also used as the boundary condition of heat transfer in the heat treatment processes. The HTC obtained for unagitated oil is used only for application to vertical surfaces. HTC data for agitated oil may be entered into the simulation models for outer surface regardless of the location of surfaces.

The results showed that the effect of oil agitation on the cooling process was reflected in the vapour phase and a significant influence of agitation in the convective heat transfer. The use of HTC for agitated oil is suitable for numerical experiments through software SYSWELD or DEFORM and of course for real experiments in the heat treatment process.

\section{REFERENCES}

[1] The Heat Treatment Simulation Solution from ESI GROUP (2011), from http://www.esigmbh.de/ downloads/ESI/Dokumente/Welding/old/The_Heat_ Treatment_Solution_Overview_180306.pdf, accessed on 2011-11-18.

[2] Troell, E., Kristoffersen, H., Bodin, J., Segerberg, S., Felde, I. (2007). Unique software bridges the gap between cooling curves and the result of hardening. Carl Hanser Verlag, München, p. 110-115.

[3] Bodin, J., Segerberg, S., Lövgren, M. (2005). IVF SmartQuench to ensure the reliability of the coolant, from http://extra.ivf.se/smartquench/articles_and_lit. asp, accessed on 2011-11-18.

[4] Taraba, B., Španielka, J. (2010). Combined heat transfer coefficient calculation for cooled probe to 850 ${ }^{\circ} \mathrm{C}$ in quenching oil. The international conference of the Carpathian Euro-region specialists in industrial system, p. 281-286.

[5] Petrofer Hildesmein (2011), from http://www.norteks. ru/en/product/petrofer/branded_items/harden_comp/, accessed on 2011-11-18.

[6] Totten, G.E., Werster, G.M., Tensi, H.M., Liscic, B. (1997). Standards for Cooling Curve Analysis of Quenchants. Heat Treatment of Metals, vol. 4, p. 92-94

[7] Ansys Theoretical Manual (2011), from http://www. pdfqueen.com/pdf/an/ansys-10-users-manual/10/, accessed on 2011-11-18.

[8] Incropera, F.P., Dewitt, D. (1996). Fundamentals of heat and mass transfer. John Wiley \& Sons, New York. 